\title{
QUALITATIVE ANALYSIS OF THE COMPOSITION NON-VOLATILE BRAZILIAN WHOLE GRAPE JUICES USING QEXACTIVETM (HYBRID QUADRUPOLE-ORBITRAP MASS SPECTROMETER) AND PRINCIPAL COMPONENT ANALYSIS (PCA)
}

\author{
ALVES, Andréa Aparecida Ribeiro ${ }^{1 *}$; BARBOSA, Elisabete dos Santos ${ }^{2}$ \\ ${ }^{1}$ Universidade Federal Fluminense, Instituto de Ciências Exatas, Departamento de Química, Rua \\ Desembargador Ellis Hermydio Figueira, 783, Bairro Aterrado CEP 27213-145, Volta Redonda - RJ, Brasil \\ (fone: +55 24 30768986) \\ ${ }^{2}$ Instituto Federal de Educação, ciência e tecnologia do Rio de Janeiro, Campus Nilo Peçanha - Pinheiral, Rua \\ José Breves, 550, Bairro Centro CEP27197-000, Pinheiral - RJ, Brasil \\ (fone: +552433568200 ) \\ ${ }^{*}$ Autor correspondente \\ e-mail: aaralves@id.uff.br \\ Received 18 August 2017; received in revised form 02 October 2017; accepted 03 October 2017

\section{RESUMO} \\ Os constituintes majoritários do suco de uva são os compostos não-voláteis e são responsáveis, na \\ maioria das vezes, pelas características organolépticas do produto, em particular o sabor e a coloração, \\ atributos associados a qualidade e aceitação do produto pelos consumidores. $O$ objetivo deste artigo foi \\ estudar, de forma qualitativa, os compostos não-voláteis dos sucos de uva integrais brasileiros utilizando o \\ QExactive $^{T M}$ - Hybrid Quadrupole-Orbitrap Mass Spectrometer e análise de componentes principais (PCA). \\ Com o QExactive ${ }^{T M}$, equipamento de alta resolução e exatidão, foi possível identificar ácidos orgânicos, ácidos \\ fenólicos e alguns aminoácidos nas amostras. A PCA mostrou-se uma ferramenta importante, pois através \\ dela, foi possível obter informações quanto ao agrupamento dos sucos estudados correlacionando-os com os \\ compostos identificados no QExactive ${ }^{T M}$.
}

Palavras-chave: grupos químicos, bebida, cor e sabor

\section{ABSTRACT}

The main constituents of grape juice are non-volatile compounds and are responsible, in most cases, the organoleptic characteristics of the product, particularly flavor and color, attributes related to quality and acceptance product by consumers. The aim of this paper was to study, in a qualitative way, the non-volatile compounds of Brazilian whole grape juices using QExactive TM (Hybrid Quadrupole-Orbitrap Mass Spectrometer) and principal component analysis (PCA). With QExactive ${ }^{\mathrm{TM}}$, high resolution and precision equipment, it was possible to identify organic acids, phenolic acids and certain amino acids in the samples. The PCA proved to be an important tool, because through it, it was possible to obtain information about the group juices studied correlating them with the compounds identified in QExactive ${ }^{\mathrm{TM}}$.

Keywords: chemical groups, drink, color and flavour 


\section{INTRODUÇÃO}

Os sucos de uva possuem compostos majoritários e não-voláteis que são açúcares, ácidos orgânicos, vitaminas, pectina, compostos fenólicos entre outros, e estes são responsáveis, na maioria das vezes, pela coloração e sabor da bebida (Catharino et al., 2006), e estão diretamente vinculadas a critérios de qualidade e aceitação do produto pelos consumidores.

$\mathrm{Na}$ casca da uva encontram-se metabólitos secundários como antocianinas (cianidina, peonidina), flavonóis, estilbenos (resveratrol) e os ácidos orgânicos (tartárico, málico, cítrico), nas sementes estão os flavonóis (taninos, catequina, epicatequina) (Fuleki e Silva, 2003; Koyama et al., 2007) e nelas também há grande quantidade de fibra e óleo, e na polpa, os compostos fenólicos são os mais abundantes (Cheynier, 2005).

$\mathrm{Na}$ produção dos sucos tintos a polpa é aquecida com a casca e a semente resultando em maior incorporação de compostos fenólicos - além da obtenção de cor intensa destes sucos - quando comparados aos sucos brancos (Fuleki e Silva, 2003; Dani et al., 2007).

Algumas técnicas já foram utilizadas para analisar compostos não-voláteis em uvas, vinhos e sucos de uvas. Fenóis, flavonóides e açúcares foram identificados em amostras de mosto (uva em fermentação) provenientes de seis variedades de uvas cultivadas no Brasil Vitis (V.) vinifera: cultivar (cv.) Pinot Noir, Cabernet Sauvignon, Malbec, Merlot, e V. Labrusca: cv. Isabel, Bordô, de procedência do Rio Grande do Sul, utilizando a espectrometria de massas por ionização com eletrospray e analisador tempo de voo (ESI-TOF/MS) (Catharino et al., 2006).

Sucos de uva da cultivar italiana Falanghina (Vitis vinifera), foram analisados usando cromatografia líquida e espectrometria de massas com ionização por electrospray e analisador quadrupolo (CL/ESI-MS, em modo negativo) para detectar marcadores de tipicidade e autenticidade (Nasi et al., 2008). Os autores realizaram extração em fase sólida (EFS) para reduzir interferentes, e detectaram terpenos glicosilados presentes em baixas concentrações nas uvas.

O uso de equipamentos com tecnologia avançada promovem um diferencial nas pesquisas e proporciona resultados mais precisos e confiáveis. $\mathrm{O}$ equipamento QExactive $^{T M}$ Hybrid Quadrupole-Orbitrap Mass Spectrometer) possui alto poder de resolução
(140.000) e excelente exatidão em massa (até a quinta casa decimal), reduzindo significativamente falsas identificações (Iglesias, 2015). O processamento dos dados é semelhante ao utilizado na Espectrometria de Massa com transformada de Fourier de íons de ressonância ciclotrônica (FT-ICR/MS), onde todos os íons são detectados simultaneamente durante um período de tempo determinado e a resolução pode ser melhorada pelo aumento da força do campo ou do aumento da duração de detecção (Makarov et al., 2006a, (Makarov et al., 2006b)

O objetivo deste artigo foi estudar, de forma qualitativa, a composição não-volátil dos sucos de uva integrais brasileiros, utilizando o QExactive $^{T M}$ e com 0 uso da análise de componentes principais (PCA) analisar e correlacionar os sucos estudados com os compostos identificados no QExactive ${ }^{T M}$.

\section{PARTE EXPERIMENTAL}

\subsection{Material}

Foram adquiridos para este trabalho, 33 sucos de uva comerciais e experimentais, que estão listados na Tabela $1 \mathrm{com}$ suas especificações (procedência do suco, tipo, natureza, cultivar e espécie da uva), todos da safra 2012, sendo os comerciais obtidos em supermercados de Niterói - RJ (Brasil) e os experimentais foram obtidos de empresas parceiras, EMBRAPA Semiárido e EPAMIG.

Os solventes utilizados foram: acetona, metanol e solução de $\mathrm{NH}_{4} \mathrm{OH}$ (concentração 1 $\mathrm{mg} \mathrm{L}^{-1}$ ), adquiridos da Tedia Brasil.

Para o cleanup dos sucos foi utilizado cartucho EFS Florisil (marca J. T. Baker ${ }^{\circledR}$, USA), contendo $100 \mathrm{mg}$ de adsorvente/cartucho. Filtros Millipore ${ }^{\circledR}$ (Membrana Fluoropore em PTFE, 0,2 $\mu \mathrm{m}$ de Poro, $25 \mathrm{~mm}$, Corpo em PE).

\subsection{Condições de análise}

Foi utilizado um espectrômetro de massas de alta resolução QExactive ${ }^{T M}$ (Thermo Scientific, Bremen, Alemanha) como ionização por electrospray (ESI) com modo de aquisição negativo e positivo concomitantemente (um diferencial deste equipamento). A aquisição foi realizada no modo Full MS em resolução de 140.000, AGC target de $10^{6}$, tempo máximo de injeção de $200 \mathrm{~ms}$ e intervalo de $\mathrm{m} / \mathrm{z}$ de 50 a 750. A amostra foi injetada em um sistema 
Accela 1250 Pump acoplado a um injetor automático Accela Open (Thermo Scientific, San Jose, EUA).

As amostras foram injetados em coluna C18 Thermo Scientific $(2.1 \times 50 \mathrm{~mm}, 1.9 \mu \mathrm{m})$, em corrida cromatográfica com fluxo constante de 50 $\mu \mathrm{L} \mathrm{min}{ }^{-1}$, em um sistema isocrático com $100 \%$ de acetonitrila, num tempo total de corrida de $5 \mathrm{~min}$. A sequência de corridas e configurações foram controladas pelo software Xcalibur $^{\mathrm{TM}}$, 0 tratamento dos dados também foi realizado neste software.

\subsection{Preparo dos extratos dos sucos para injeção no QExactive ${ }^{T M}$}

Para o preparo dos extratos dos sucos utilizou-se $2 \mathrm{~mL}$ de suco de uva. O cartucho de EFS Florisil foi acondicionado com $1 \mathrm{~mL}$ de acetona, e em seguida foi adicionado o suco de uva, que foi eluído com $2 \mathrm{~mL}$ de metanol. O eluato foi levado à secura sob fluxo de $N_{2}$, e então ressuspendido com $2 \mathrm{~mL}$ de metanol. Desse extrato de suco, retirou-se uma alíquota de $50 \mu \mathrm{L}$, ao qual se adicionou $950 \mu \mathrm{L}$ da solução de $\mathrm{CH}_{3} \mathrm{OH} / \mathrm{H}_{2} \mathrm{O} / \mathrm{NH}_{4} \mathrm{OH}$ (solução $1 \%$ ). Esta solução final foi colocada em filtro de $0,2 \mu \mathrm{m}$ da Millipore ${ }^{\circledR}$, e então foi recolhido em um vial apropriado para injeção no QExactive ${ }^{T M}$ e assim foram injetados $20 \mu \mathrm{L}$ desta solução final, em fluxo de $50 \mu \mathrm{L} \min ^{-1}$ e injeção automática. Procedeu-se os procedimentos supracitados para os 33 sucos em triplicata de extração.

Para o tratamento dos dados fez-se uma busca na literatura e montou-se uma tabelareferência com informações dos compostos nãovoláteis, como massa/carga $(\mathrm{m} / \mathrm{z})$, tipo de equipamento e principais fragmentos para uvas e seus derivados. Logo após, realizou-se a análise de cada suco utilizando o software $\mathrm{X}_{\text {calibur }}{ }^{\mathrm{TM}}$ que forneceu o espectros ESI-MS, com valores de $\mathrm{m} / \mathrm{z}$ experimental $\left(\mathrm{m} / \mathrm{z}_{\text {exp. }}\right.$ ) com cinco casas decimais, obtendo-se a abundância da área relativa desses íons. Em seguida, foram realizadas comparações das informações da tabela-referência com os valores obtidos pelo software $\mathrm{X}$ calibur ${ }^{\mathrm{TM}}$. A verificação da razão massa/carga teórica $\left(\mathrm{m} / \mathrm{z}_{\text {teor. }}\right)$ foi obtida no próprio software através da fórmula molecular da substância identificada, e assim foi realizado o cálculo do erro, em ppm, segundo a equação 1 (Eq. 1) retirada de Iglesias (2015):

$$
E(\text { ppm })=\left|\left(m / z_{\text {exp. }}-m / z_{\text {teor. }}\right) /\left(m / z_{\text {exp. }}\right)\right| \times 10^{6}(\text { Eq. } 1)
$$

Com os valores de área obtidos no tratamento dos dados das injeções no QExactive $^{T M}$ foi possível fazer a PCA dos compostos não-voláteis dos sucos de uva e tentar agrupá-los utilizando o programa SPSS_17 Statistics.

\section{RESULTADOS E DISCUSSÃO:}

Neste trabalho, fez-se inicialmente um cleanup da amostra utilizando EFS Florisil, pois esta técnica apresentou resultados eficientes tanto para cleanup quanto para pré-concentração de analitos em matrizes alimentares (Alves et al., 2012; Alves et al., 2014). As soluções finais, obtidas dos extratos de suco de uva integral, utilizando a EFS Florisil, foram filtrados em filtro Millipore ${ }^{\circledR}$, para a retirada de possíveis resíduos insolúveis presentes na solução final, uma vez que estes podem entupir a seringa de injeção e prejudicar 0 funcionamento do QExactive ${ }^{T M}$ (Makarov, 2006a).

Os dados foram tratados utilizando do software Xcalibur ${ }^{\mathrm{TM}}$, obtendo-se um perfil dos compostos não-voláteis dos sucos, como observa-se na Tabela 2, onde estão o nome das substâncias, suas respectivas fórmulas moleculares e os valores de massa/carga $(\mathrm{m} / \mathrm{z})$ experimental (exp.) e teórico (teor.), além do erro $\mathrm{E}(\mathrm{ppm})$ relativo às $m / z_{\text {exp. }}$ e $m / z_{\text {teor. }}$ para verificação da confiabilidade da identificação dos mesmos. O erro indica o quanto os valores obtidos se destoaram dos "verdadeiros" valores de $m / z$, logo para este tipo de equipamento, que possui alta resolução em massa exata, o erro deve ser inferior a 10 ppm (Iglesias, 2015), logo observa-se que os valores de $E$ (ppm) obtidos variaram de 0,42 a 8,06 ppm, sendo assim considerados satisfatórios.

$\mathrm{Na}$ Tabela 3 encontram-se os valores da abundância (média das áreas da triplicata de cada suco) das substâncias mencionadas na Tabela 2, presentes nos sucos de uva trabalhados. Foram calculados com base nestas áreas, os coeficientes de variação (CV \%), os quais variaram de 0,8 a $6,8 \%$, apresentando baixa dispersão dos valores, uma vez que valores de CV \% até $15 \%$ é considerado aceitável quando se trata de matrizes complexas (Miller e Miller, 1988; EMEA, 2015).

Observa-se que em todos os sucos foram identificados o ácido fosfórico, e os ácidos tartárico, málico, cítrico e ascórbico (exceção do suco $A L)$, já mencionados em uvas híbridas ( $V$. 
vinifera com $V$. labrusca) para elaboração de vinhos (Biasoto et al., 2010). O pterostilbeno, encontrado nos sucos comerciais CB, CM, DC, $E P, \quad M V, P G$ e SP, e nos experimentais SA_chard_B e SA mosc_B, é um estilbeno, e assim como o resveratrol é produzido pelas uvas para o combate a fungos e vírus; e possui propriedades antiinflamatórias e antioxidantes quando ingeridas por animais (Langcake e Pryce, 1977).

O ácido clorogênico (ACG), aqui representado pelo ácido 4-O-cafeoilquinico foi identificado apenas no suco experimental SA coraB e trata-se de um éster polifenólico encontrado em vegetais e frutas, principalmente no café. O ACG 5-O-cafeoilquinico, no entanto foi observado em dezoito tipos de sucos, sendo encontrado principalmente em frutas. Estudo mostra que 0 ácido cafeoilquinico já foi identificado em sucos de uva da cv. Concord ( $V$. labrusca) produzidos em Farroupilha, RS (Oliveira et al., 2013). O ACG atua em diversos sistemas biológicos, nas atividades antimutagênica, antitumoral, no controle da obesidade, analgésica, antipirética, ansiolítica, anti-microbicida, antifúngica, antiviral, antioxidante e anti-inflamatória (Clifford, 2000).

O ácido sinápico é derivado do ácido cinâmico e possui atividade antioxidante, assim como o ácido vanílico, que é derivado do ácido benzóico (Ramalho e Jorge, 2006), sendo encontrados respectivamente nos sucos SA_coraFM, GA, GB, SA_isaB, $S A$ isaM, SA_mosc_B, PZ, SP). O manitol, por sua vez, é um açúcar obtido da frutose, possui atividade diurética e é utilizado em alimentos dietéticos (Cruz et al., 2004). Pode ser utilizado como adoçante, e foi identificado apenas no suco SB, um suco orgânico e comercial.

O L-triptofano é um aminoácido que auxilia na síntese da serotonina e está associado ao bem-estar, sendo importante no tratamento e prevenção da depressão e ansiedade, e pode ser encontrado em alguns alimentos (Correia et al., 2014), sendo observado nos sucos GD, GD_B, SA_isaM, CB, SA_coraFM, SA_coraM e UV. Outro aminoácido, a $L$-arginina, foi identificada nos sucos PZ e SA_chard_B, sendo responsável pela cicatrização de feridas, antitumoral, e combinada com a lisina, reduzem a ansiedade; a L-tirosina, ajuda na produção de outros hormônios, que melhoram o estado de alerta e a alegria (Correia et al., 2014), observada nos sucos AL, AM, AU, SA_chard_B, CL e CM.
$\mathrm{Na}$ Tabela 4 encontram-se os códigos dos compostos para a análise dos componentes principais (PCA), cujas áreas se encontram descritas na Tabela 3. Alguns compostos da Tabela 3 estão ausentes na Tabela 4, pois seus valores não foram significativos para auxiliar na separação dos sucos na PCA.

$\mathrm{Na}$ Figura 1 encontra-se a análise de componentes principais (PCA) para os sucos de uva tintos (vide códigos na Tabela 1), onde se observa o gráfico de scores (A) dos sucos de uva e o gráfico de loadings (B) com os compostos químicos identificados (vide códigos na Tabela 4). As duas componentes principais descrevem $73,7 \%$, promovendo boa separação e/ou agrupamento das amostras.

Os sucos SI e UV estão relacionados com os compostos C34 (L-tirosina), C35 (galato de metila), C40 (L-leucina), C48 (L-triptofano) e C50 (pterostilbeno), o que mostra que há maior quantidade destes compostos nestes sucos, conseguindo separá-los, mesmo que pouco dos demais sucos. O galato de metila é um composto fenólico encontrado no vinho e no gerânio (Geranium niveum) (Calzada et al., 1999).

Pode-se observar no gráfico de scores, Figura $1 \mathrm{~A}$, que há um grupo de sucos à esquerda, sendo 3 sucos experimentais (SA) e 8 sucos comerciais. Nota-se que, no gráfico de loadings, Figura 1B, não há correlação direta com nenhuma substância. Esse fato informa que nenhuma substância da Tabela 4 foi eficaz para isolá-los.

Os sucos AL e AU apresentam correlação com os compostos C8 (ácido ascórbico), C23 (Lvalina), C25 (malonato de dietila), C28 (ciscoumárico), C36 (ácido propiônico) e C37 (5-Ocafeoilquinico). Vale ressaltar que 0 ácido ascórbico (vitamina C) é uma furanolactona muito importante na dieta humana, é um poderoso antioxidante, sendo usado para transformar os radicais livres de oxigênio em formas inertes, e pode ser encontrado em várias frutas e legumes (Nelson e Cox, 2012).

Já os sucos $P Z$ e GB possuem 11 compostos propostos que ajudam a separá-los do grupo maior. Os compostos C31 (ácido siríngico), C44 (resveratrol), C56 (manitol), C58 (4-O-cafeoilquinico) e C59 (ácido sinápico), correlaciona-se a 8 sucos, deixando-os agrupados entre si e separados dos demais grupos. O ácido siringico é derivado do ácido gálico e está presente no vinho tinto e nas cascas das uvas vermelhas possui atividade antioxidante (Gálvez et al., 1994). 
Os compostos sem marcação são aqueles que podem estar relacionados com os sucos $P Z$ e GB, na região inferior do gráfico de loadings e com os sucos $A L$ e $A U$ na parte superior.

A PCA foi utilizada para caracterização de vinhos elaborados com uvas híbridas ( $V$. vinifera com $V$. labrusca) e observaram, de maneira geral, que a PCA estaria correlacionando o parâmetros de acidez de uma amostra com a presença do ácido succínico, e em outra amostra, correlacionava com o ácido ascórbico que conferiria carater amargo. Os autores propuseram um potencial uso destas informações para auxiliar as vinícolas no controle de qualidade (Biasoto et al., 2010).

$\mathrm{Na}$ Figura 2 encontra-se a PCA para os sucos brancos, onde se observa o gráfico de scores (A) dos sucos de uva e o gráfico de loadings (B) com os compostos químicos propostos, sendo que as componentes principais descreveram 66,0 \%, conferindo separação das amostras.

Embora pequeno o número de amostras, observa-se que os sucos estão bem separados, com exceção dos sucos SA_mosc_B e GD_B, que se agruparam por influência dos compostos C40 (L-leucina), C48 (L-triptofano) e C50 (pterostilbeno), compostos que foram igualmente observados nos sucos tintos SI e UV.

$O$ suco experimental SA chard $B$ do gráfico de scores, Figura $2 \mathrm{~A}$, correlaciona-se no gráfico de loadings (Figura 2B) com os compostos C5 (ácido succínico) e C54 (arginina), e o suco CM_B não possui nenhuma substância correlacionada a ele. No entanto, no gráfico de loadings observa-se grande número de compostos sem correlação com nenhum suco estudado, o que pode estar relacionado ao baixo número de amostras.

O ácido trans-caftárico (C30) é um composto fenólico não-flavonoídico encontrado na uva, sendo responsável pelo tom amarelado dos vinhos e sucos brancos (Lee e Jaworski.1987) O tirosol (41) é um antioxidante fenólico natural presente em uma variedade de matérias primas naturais, dentre elas a uva, o chá e óleo de oliva, é um derivado do álcool fenetílico (Giovannini et al., 1999). Os sucos SA_ita_B e PE_B possuem, respectivamente 7 e 10 substâncias que os separam dos demais sucos brancos.

A PCA se mostrou uma ferramenta importante na análise da composição do perfil não-volátil dos sucos, pois através dela foi possível correlacionar o agrupamento de alguns sucos com compostos específicos (identificados pelo QExactive ${ }^{T M}$ ), o que leva a crer que estes compostos podem ser potenciais marcadores químicos destes sucos.

\section{CONCLUSÕES:}

Conclui-se que foi possível estudar, de forma qualitativa, a composição não-volátil dos sucos de uva integrais brasileiros utilizando o equipamento QExactive ${ }^{T M}$ (Hybrid QuadrupoleOrbitrap Mass Spectrometer) que com sua alta resolução e exatidão, identificou nas amostras ácidos orgânicos, ácidos fenólicos, alguns aminoácidos, entre outros. Com esta identificação, foi possível utilizar a análise dos componentes principais (PCA), que se mostrou eficiente, pois foi possível agrupar alguns sucos de uva correlacionando-os com compostos nãovoláteis comum entre eles.

\section{AGRADECIMENTOS:}

As autoras agradecem a Claudia $M$. de Rezende do IQ/UFRJ, Giuliano E. Pereira da EMBRAPA Semiárido, Rafael Garrett do IQ/UFRJ pelo apoio; à Capes, CNPq e à FAPERJ pelo apoio financeiro; à EMBRAPA Semiárido e à EPAMIG pelas amostras; ao Henrique Marcelo G. Pereira e Vinícius F. Sardela do LBCD-LADETEC/IQ-UFRJ pela utilização do equipamento $Q$ Exactive $^{T M}$.

\section{REFERÊNCIAS:}

1. Alves, A.A.R.; Rezende, M.J.C.; Hovell, A.M.C.; Bizzo, H.R.; Oliveira, A.C.L.; Rodrigues, S.V.; Rezende, C.M. Journal of the Brazilian Chemical Society. 2012, 23, 306.

2. Alves, A.A.R.; Rodrigues, A.S.; Paula Barros, E.B.; Uekane, T.M.; Bizzo, H.R.; Rezende, C.M. Food Analytical Methods. 2014, 7, 1834. 
3. Biasoto, A.C.T.; Catharino, R.R.; Sanvido, G.B.; Eberlin, M.N.; Silva, M.A.A.P. Food Quality and Preference 2010, 21, 755.

4. Calzada, F.; Cerda-García-Rojas, C.M.; Meckes, M.; Cedillo-Rivera, R.; Bye, R.; Mata, R. Journal of Natural Products. 1999, 62, 705.

5. Catharino, R.R.; Cunha, I.B.S.; Fogaça, A.O.; Facco, E.M.P.; Godoy, H.T.; Daudt, C.E.; Eberlin, M.N.; Sawaya, A.C.H.F. Journal of Mass Spectrometry. 2006, 41, 185.

6. Cheynier, V. American Journal of Clinical Nutrition. 2005, 81S, $223 \mathrm{~S}$.

7. Clifford, M.N. Journal of the Science of Food and Agriculture. 2000, 80, 1033.

8. Correia, M.I.T.D.; Quirino, I.E.P. Nutrição e vida. 2014, 1.

9. Cruz, J.; Minoja, G.; Okuchi, K.; Facco, E. Journal of Neurosurgery. 2004, 100, 376.

10. Dani, C.; Oliboni, L.S.; Vanderlinde, R.; Bonatto, D.; Salvador, M.; Henriques, J. A. P. Food and Chemical Toxicolgy. 2007, 45, 2574.

11. EMEA - European Medicines Agency. http://www.ema.europa.eu/docs/en_GB/d ocument_library/Scientific_guideline/2009/ 09/WC500002662.pdf. Acessada em: novembro 2016.

12. Fuleki, T.; SILVA, J.M. Journal of Agricultural and Food Chemistry. 2003, 51,640 .

13. Gálvez, M.C.; Barroso, C.G.; PérezBustamante, J.A. Zeitschrift für Lebensmitteluntersuchung und Forschung A. 1994, 199, 29.

14. Giovannini, C.; Straface, E.; Modesti, D.; Coni, E.; Cantafora, A.; De Vincenzi, M.; Malorni, W.; Masella, R. Journal of Nutrition. 1999, 129, 1269.

15. Iglesias, A.H. http://www.cnpsa.embrapa.br/met/images/ arquivos/17MET/minicursoamadeu- iglesias.pdf. Acessada em novembro 2015.

16. Koyama, K.; Goto-Yamamoto, N.; Hashizume, K. Bioscience Biotechnology and Biochemistry. 2007, 71, 958.

17. Langcake, P.; Pryce, R. J.. Experientia. 1977, 33, 151.

18. Lee, C. Y.; Jaworski, A. American Journal of Enology and Viticulture. 1987, 38, 277.

19. Makarov, A.; Denisov, E.; Kholomeev, A.; Balschun, W.; Lange, O.; Strupat, K.; Horning, S. Analytical Chemistry. 2006a, $78,2113$.

20. Makarov, A.; Denisov, E.; Lange, O.; Strupat, K.; Horning, S. Journal of the American Society for Mass Spectrometry 2006b, 17, 977.

21. Miller, J. C.; Miller, J. N. Statistics for Analytical Chemistry, 2 $2^{\underline{a}}$ ed., Ellis Horwood: Chichester, 1988.

22. Nasi, A.; Ferranti, P.; Amato, S.; Chianese, L. Food Chemistry. 2008, 110, 762.

23. Nelson, D.L.; Cox, M.M. Lehninger Principles of Biochemistry, 6 $6^{\mathrm{a}}$ ed., W. H. Freeman, 2012.

24. Oliveira, D.N.; Sartor, S.B.; Damário, N.; Göllucke, A.P.B.; Catharino, R.R. Journal of Food Measurement and Characterization. 2013, 8, 9.

25. Ramalho, V.C.; Jorge, N. Química Nova. 2006, 29, 755. 
Tabela 1. Relação dos sucos de uva integrais com suas especificações: procedência do suco, tipo, natureza, cultivar e espécie da uva.

\begin{tabular}{|c|c|c|c|c|c|}
\hline $\begin{array}{l}\text { Código do } \\
\text { suco }\end{array}$ & Procedência ${ }^{a}$ & Tipo & Natureza & $\begin{array}{c}\text { Cultivar da } \\
\text { uva }\end{array}$ & Espécie da uva \\
\hline AM & Rio Grande do Sul & tinto & Comercial & Isabel/bordô & Vitis labrusca \\
\hline$A L$ & Rio Grande do Sul & tinto & Comercial & Isabel/bordô & Vitis labrusca \\
\hline$A U$ & Rio Grande do Sul & tinto & Comercial & Isabel/bordô & Vitis labrusca \\
\hline CB & Rio Grande do Sul & tinto & Comercial & Isabel/bordô & Vitis labrusca \\
\hline $\mathrm{CL}$ & Rio Grande do Sul & tinto & Comercial & Isabel/bordô & Vitis labrusca \\
\hline $\mathrm{CM}$ & Rio Grande do Sul & tinto & Comercial & Isabel/bordô & Vitis labrusca \\
\hline $\mathrm{DC}^{*}$ & Rio Grande do Sul & tinto & Comercial & Isabel/bordô & Vitis labrusca \\
\hline DG & Rio Grande do Sul & tinto & Comercial & Isabel/bordô & Vitis labrusca \\
\hline $\mathrm{GA}$ & Rio Grande do Sul & tinto & Comercial & Isabel/bordô & Vitis labrusca \\
\hline GB & Rio Grande do Sul & tinto & Comercial & Isabel/bordô & Vitis labrusca \\
\hline $\mathrm{GD}^{*}$ & Rio Grande do Sul & tinto & Comercial & Isabel/bordô & Vitis labrusca \\
\hline MT & Rio Grande do Sul & tinto & Comercial & Isabel/bordô & Vitis labrusca \\
\hline MV & Rio Grande do Sul & tinto & Comercial & Isabel/bordô & Vitis labrusca \\
\hline PE & Rio Grande do Sul & tinto & Comercial & Isabel/bordô & Vitis labrusca \\
\hline$P \bar{G}$ & Rio Grande do Sul & tinto & Comercial & Isabel/bordô & Vitis labrusca \\
\hline PZ & Rio Grande do Sul & tinto & Comercial & Isabel/bordô & Vitis labrusca \\
\hline $\mathrm{SB}^{*}$ & São Paulo & tinto & Comercial & Isabel/bordô & Vitis labrusca \\
\hline SI & Rio Grande do Sul & tinto & Comercial & Isabel/bordô & Vitis labrusca \\
\hline SN & Rio Grande do Sul & tinto & Comercial & Isabel/bordô & Vitis labrusca \\
\hline $\mathrm{SP}^{*}$ & Rio Grande do Sul & tinto & Comercial & Isabel/bordô & Vitis labrusca \\
\hline US & Rio Grande do Sul & tinto & Comercial & Isabel/bordô & Vitis labrusca \\
\hline$E P^{*}$ & Minas Gerais & tinto & Experimental & Isabel/bordô & Vitis labrusca \\
\hline SA_isaM & Pernambuco & tinto & Experimental & Isabel & Vitis labrusca \\
\hline SA_isaB & Pernambuco & tinto & Experimental & Isabel & Vitis labrusca \\
\hline SA_coraB & Pernambuco & tinto & Experimental & BRS cora & Vitis labrusca \\
\hline SA_coraM & Pernambuco & tinto & Experimental & BRS cora & Vitis labrusca \\
\hline SA coraFM & Pernambuco & tinto & Experimental & BRS cora & Vitis labrusca \\
\hline CM_B & Rio Grande do Sul & branco & Comercial & Italia & Vitis vinífera \\
\hline GD_B* & Rio Grande do Sul & branco & Comercial & Italia & Vitis vinífera \\
\hline PE B & Rio Grande do Sul & branco & Comercial & Italia & Vitis vinífera \\
\hline SA chard B & Pernambuco & branco & Experimental & Chardonnay & Vitis vinífera \\
\hline SA_mosc B & Pernambuco & branco & Experimental & Moscato & Vitis vinífera \\
\hline SA ita $\bar{B}$ & Pernambuco & branco & Experimental & Itália & Vitis vinífera \\
\hline
\end{tabular}


Tabela 2. Compostos não-voláteis presentes nos sucos de uva, fórmula molecular, $m / z_{\text {exp }}$ (experimental) e $\mathrm{m} / \mathrm{z}_{\text {teor. }}$ (teórico), erro $E(\mathrm{ppm})$ obtidos na análise por QExactive ${ }^{T M}$

\begin{tabular}{|c|c|c|c|c|}
\hline Substância & Fórmula & $m / z_{\text {exp. }}$ & $m / z_{\text {teor. }}$ & $E$ (ppm) \\
\hline acido fosfórico & $\mathrm{H}_{3} \mathrm{PO}_{4}$ & 96,95871 & 96,95915 & 4,53 \\
\hline acido tartárico & $\mathrm{C}_{4} \mathrm{H}_{6} \mathrm{O}_{6}$ & 149,00797 & 149,00789 & 0,54 \\
\hline acido málico & $\mathrm{C}_{4} \mathrm{H}_{6} \mathrm{O}_{5}$ & 133,01296 & 133,01251 & 3,38 \\
\hline acido cítrico & $\mathrm{C}_{6} \mathrm{H}_{8} \mathrm{O}_{7}$ & 191,01890 & 191,01795 & 4,97 \\
\hline acido succínico & $\mathrm{C}_{4} \mathrm{H}_{6} \mathrm{O}_{4}$ & 117,01805 & 117,01756 & 4,18 \\
\hline acido acético & $\mathrm{C}_{2} \mathrm{H}_{4} \mathrm{O}_{2}$ & 59,01251 & 59,01258 & 1,18 \\
\hline acido ascórbico & $\mathrm{C}_{6} \mathrm{H}_{8} \mathrm{O}_{6}$ & 176,13380 & 176,13456 & 4,31 \\
\hline ácido $p$-coumarico & $\mathrm{C}_{9} \mathrm{H}_{8} \mathrm{O}_{3}$ & 165,09284 & 165,09277 & 0,42 \\
\hline ácido cafeico & $\mathrm{C}_{9} \mathrm{H}_{8} \mathrm{O}_{4}$ & 180,05851 & 180,05871 & 1,11 \\
\hline ácido quinico & $\mathrm{C}_{7} \mathrm{H}_{12} \mathrm{O}_{6}$ & 191,01890 & 191,01834 & 2,93 \\
\hline ácido trans-fertarico & $\mathrm{C}_{14} \mathrm{H}_{14} \mathrm{O}_{9}$ & 325,09284 & 325,09272 & 0,37 \\
\hline ácido ferúlico & $\mathrm{C}_{10} \mathrm{H}_{10} \mathrm{O}_{4}$ & 193,03455 & 193,03536 & 4,19 \\
\hline ácido aspártico & $\mathrm{C}_{4} \mathrm{H}_{7} \mathrm{NO}_{4}$ & 134,01636 & 134,01696 & 4,47 \\
\hline ácido malonico & $\mathrm{C}_{3} \mathrm{H}_{4} \mathrm{O}_{4}$ & 102,97443 & 102,97391 & 5,05 \\
\hline ácido siringico & $\mathrm{C}_{9} \mathrm{H}_{10} \mathrm{O}_{5}$ & 199,80428 & 199,80370 & 2,90 \\
\hline ácido propionico & $\mathrm{C}_{3} \mathrm{H}_{6} \mathrm{O}_{2}$ & 72,99172 & 72,99201 & 3,97 \\
\hline acido galico & $\mathrm{C}_{7} \mathrm{H}_{6} \mathrm{O}_{5}$ & 170,09014 & 170,09097 & 4,87 \\
\hline ácido cinâmico & $\mathrm{C}_{9} \mathrm{H}_{8} \mathrm{O}_{2}$ & 149,04439 & 149,04508 & 4,62 \\
\hline acido carbônico & $\mathrm{H}_{2} \mathrm{CO}_{3}$ & 59,91258 & 59,91296 & 6,34 \\
\hline ácido sinapico & $\mathrm{C}_{11} \mathrm{H}_{12} \mathrm{O}_{5}$ & 225,00701 & 225,00786 & 3,78 \\
\hline ácido vanilico & $\mathrm{C}_{8} \mathrm{H}_{8} \mathrm{O}_{4}$ & 168,98842 & 168,98855 & 0,77 \\
\hline ácido coutarico & $\mathrm{C}_{13} \mathrm{H}_{12} \mathrm{O}_{8}$ & 295,06708 & 295,06781 & 2,47 \\
\hline ácido trans-caftárico & $\mathrm{C}_{13} \mathrm{H}_{12} \mathrm{O}_{9}$ & 311,16867 & 311,16758 & 3,50 \\
\hline ácido cis-4-coumarico & $\mathrm{C}_{9} \mathrm{H}_{8} \mathrm{O}_{3}$ & 165,06708 & 165,06782 & 4,48 \\
\hline$L$ - asparagina & $\mathrm{C}_{4} \mathrm{H}_{8} \mathrm{~N}_{2} \mathrm{O}_{3}$ & 133,04936 & 133,04961 & 1,88 \\
\hline$L$ - glutamina & $\mathrm{C}_{5} \mathrm{H}_{10} \mathrm{~N}_{2} \mathrm{O}_{3}$ & 147,02875 & 147,02833 & 2,85 \\
\hline$L$ - lisina & $\mathrm{C}_{6} \mathrm{H}_{14} \mathrm{~N}_{2} \mathrm{O}_{2}$ & 147,02875 & 147,02909 & 2,31 \\
\hline$L-$ valina & $\mathrm{C}_{5} \mathrm{H}_{11} \mathrm{NO}_{2}$ & 118,04082 & 118,04140 & 4,91 \\
\hline$D$ - glucose & $\mathrm{C}_{6} \mathrm{H}_{12} \mathrm{O}_{6}$ & 179,05515 & 179,05484 & 1,73 \\
\hline$L$ - prolina & $\mathrm{C}_{5} \mathrm{H}_{9} \mathrm{NO}_{2}$ & 115,91945 & 115,91891 & 4,65 \\
\hline$L$ - metionina & $\mathrm{C}_{5} \mathrm{H}_{11} \mathrm{NO}_{2} \mathrm{~S}$ & 150,01137 & 150,01150 & 1,13 \\
\hline$L-$ tirosina & $\mathrm{C}_{9} \mathrm{H}_{11} \mathrm{NO}_{3}$ & 182,08775 & 182,08694 & 4,45 \\
\hline$L-$ leucina & $\mathrm{C}_{6} \mathrm{H}_{13} \mathrm{NO}_{2}$ & 132,36670 & 132,36605 & 4,91 \\
\hline L-triptofano & $\mathrm{C}_{11} \mathrm{H}_{12} \mathrm{~N}_{2} \mathrm{O}_{2}$ & 205,03484 & 205,03498 & 0,68 \\
\hline$L$ - arginina & $\mathrm{C}_{6} \mathrm{H}_{14} \mathrm{~N}_{4} \mathrm{O}_{2}$ & 175,06026 & 175,06101 & 4,28 \\
\hline 4-O-cafeoilquinico & $\mathrm{C}_{16} \mathrm{H}_{18} \mathrm{O}_{9}$ & 353,08800 & 353,08825 & 0,71 \\
\hline 5-O-cafeoilquinico & $\mathrm{C}_{24} \mathrm{H}_{32} \mathrm{O}_{8}$ & 447,03959 & 447,03920 & 0,87 \\
\hline resveratrol & $\mathrm{C}_{14} \mathrm{H}_{12} \mathrm{O}_{3}$ & 228,99380 & 228,99310 & 3,06 \\
\hline pteroestilbeno & $\mathrm{C}_{16} \mathrm{H}_{16} \mathrm{O}_{3}$ & 254,91680 & 254,91555 & 5,45 \\
\hline Apigenina & $\mathrm{C}_{15} \mathrm{H}_{10} \mathrm{O}_{5}$ & 269,08773 & 269,08827 & 2,01 \\
\hline catequina & $\mathrm{C}_{15} \mathrm{H}_{14} \mathrm{O}_{6}$ & 290,08415 & 290,08491 & 2,62 \\
\hline coniferina & $\mathrm{C}_{16} \mathrm{H}_{22} \mathrm{O}_{8}$ & 341,10898 & 341,10876 & 0,65 \\
\hline$p$-coumarato de etila & $\mathrm{C}_{11} \mathrm{H}_{12} \mathrm{O}_{3}$ & 191,01890 & 191,01736 & 8,06 \\
\hline galato de metila & $\mathrm{C}_{8} \mathrm{H}_{8} \mathrm{O}_{5}$ & 182,98779 & 182,98662 & 6,39 \\
\hline malonato de dietila & $\mathrm{C}_{7} \mathrm{H}_{12} \mathrm{O}_{4}$ & 160,07445 & 160,07533 & 5,49 \\
\hline Tirosol & $\mathrm{C}_{8} \mathrm{H}_{10} \mathrm{O}_{2}$ & 136,99571 & 136,99503 & 4,96 \\
\hline manitol & & 181,07084 & 181,07147 & 3,48 \\
\hline
\end{tabular}


Tabela 3. Valores da abundância (média das áreas da triplicata, $n=3$ ) de cada substância identificada nos sucos de uva

\begin{tabular}{|c|c|c|c|c|c|c|c|c|c|c|c|}
\hline \multirow{2}{*}{ Substâncias } & \multicolumn{11}{|c|}{ Abundância } \\
\hline & AL & AM & $A U$ & Св & SA_chard_B & $\mathrm{CL}$ & $\mathrm{CM}$ & CM_B & SA_coraB & SA_coraFM & SA_coraM \\
\hline acido fosforico & 230813813 & 430221206 & 40943917 & 138344603 & 167531059 & 11464848 & 18067475 & 419484011 & 26673168 & 107387797 & 82846949 \\
\hline acido tartarico & 74812156 & 358513087 & 13752693 & 45103386 & 21757269 & 1691592 & 3291786 & 15448307 & 5345973 & 8838098 & 24886112 \\
\hline acido acetico & 8053655 & 11369508 & 2974453 & 7006471 & 5937977 & 120083 & 217508 & 22947987 & 538784 & 5475400 & 4509861 \\
\hline acido carbonico & 0 & 0 & 0 & 372419 & 9321 & 991014 & 0 & 0 & 0 & 0 & 0 \\
\hline acido citrico & 32252173 & 35373422 & 11051592 & 5715724 & 8002452 & 571626 & 688940 & 98314206 & 447482 & 3855189 & 3637326 \\
\hline acido succinico & 5685627 & 4105379 & 3433648 & 6531138 & 225957467 & 47951 & 55231 & 26371164 & 0 & 4523560 & 8079073 \\
\hline ácido propiônico & 3090387 & 8207062 & 1838140 & 5434553 & 23530050 & 38046 & 47170 & 17028964 & 253032 & 4527367 & 0 \\
\hline acido ascorbico & 0 & 5401970 & 4807276 & 8569936 & 26191359 & 311271 & 336749 & 32331536 & 647841 & 7381285 & 6645177 \\
\hline acido málico & 305883547 & 369590109 & 80808147 & 66425671 & 322354471 & 3810513 & 5395816 & $1,104 \mathrm{E}+09$ & 4316080 & 472534144 & 423808939 \\
\hline acido $p$-coumarico & 26965278 & 14968433 & 1506449 & 8118461 & 3345956 & 301648 & 387093 & 60584192 & 210990 & 7958317 & 8784069 \\
\hline acido cafeico & 57260023 & 32773630 & 23420764 & 31022858 & 11248693 & 1568970 & 1757069 & 9734591 & 3340108 & 2544323 & 36019515 \\
\hline acido quinico & 10035752 & 13491333 & 1634567 & 5257515 & 3627675 & 114120 & 141520 & 11448911 & 517482 & 3855187 & 3637326 \\
\hline acido trans-fertarico & 41033746 & 18841558 & 18187104 & 13500739 & 34874363 & 1306089 & 3291786 & 97261560 & 231273 & 7958317 & 7993997 \\
\hline acido ferúlico & 32611524 & 199617065 & 1189199 & 10021397 & 35054535 & 1306089 & 3291786 & 97261560 & 259270 & 5239462 & 6769441 \\
\hline ácido aspártico & 11041352 & 15516039 & 3398724 & 15603690 & 18321619 & 583737 & 811925 & 47283544 & 0 & 20248509 & 18094089 \\
\hline ácido malônico & 2919926 & 4746021 & 1497327 & 4134408 & 66103 & 516548 & 620494 & 9856977 & 0 & 0 & 0 \\
\hline acido siringico & 61815 & 0 & 0 & 0 & 14591 & 0 & 20968 & 0 & 0 & 0 & 0 \\
\hline acido cinâmico & 0 & 0 & 666345 & 34283176 & 6151864 & 623810 & 3291786 & 15448307 & 5345974 & 2842512 & 24896427 \\
\hline acido sinapico & 0 & 0 & 0 & 0 & 0 & 0 & 0 & 0 & 0 & 3775766 & 0 \\
\hline acido galico & 0 & 0 & 3204145 & 158949 & 0 & 0 & 0 & 10690618 & 0 & 2556781 & 3952286 \\
\hline acido coutárico & 29891027 & 5103748 & 1481624 & 7179726 & 34879869 & 1691592 & 3291786 & 21722682 & 5192633 & 8838098 & 0 \\
\hline ácido trans-caftárico & 35131 & 1456627 & 21082 & 7527804 & 15839 & 0 & 28614 & 152536715 & 0 & 0 & 0 \\
\hline ácido cis-4-coumarico & 35620398 & 9329658 & 1509930 & 6194974 & 12742164 & 331047 & 378117 & 21722682 & 669940 & 4908936 & 4514726 \\
\hline$L$ - asparagina & 266874926 & 369590109 & 82367300 & 5790391 & 148987665 & 2872764 & 3531311 & 9084208 & 4316080 & 3359228 & 3012906 \\
\hline L-glutamina & 17548954 & 0 & 0 & 8227130 & 4876680 & 179648 & 471633 & 42437395 & 944657 & 9862785 & 10449571 \\
\hline$L$-lisina & 3292216 & 0 & 0 & 2987561 & 4876680 & 0 & 0 & 0 & 0 & 0 & 0 \\
\hline L-valina & 6888608 & 10689065 & 2591850 & 1602490 & 9749920 & 746626 & 95665 & 14967783 & 858060 & 4856160 & 3765114 \\
\hline$D$-glucose & 50577076 & 64195411 & 23420765 & 31022858 & 19588487 & 1568970 & 1757069 & 152536715 & 3340108 & 4053166 & 34250005 \\
\hline L-prolina & 161924 & 1555375 & 1853505 & 2511353 & 3485933 & 764326 & 950483 & 9084208 & 0 & 3359228 & 3012906 \\
\hline L- metionina & 217990300 & 6774985 & 23941 & 177999 & 4896906 & 1416833 & 2594007 & 12203662 & 226453 & 0 & 0 \\
\hline$L$ - tirosina & 75590 & 137678 & 93105,33 & 0 & 29145 & 618761 & 260206 & 0 & 0 & 0 & 0 \\
\hline$L$-arginina & 0 & 0 & 0 & 0 & 7124987 & 0 & 0 & 0 & 0 & 0 & 0 \\
\hline$L$-leucina & 0 & 0 & 32010,67 & 180864 & 0 & 243887 & 123615 & 0 & 0 & 0 & 6084275 \\
\hline L-triptofano & 0 & 0 & 0 & 2450051 & 0 & 0 & 0 & 0 & 0 & 3330285 & 2892451 \\
\hline 4-O-cafeoilquinico & 0 & 0 & 0 & 0 & 0 & 0 & 0 & 0 & 1327427 & 0 & 0 \\
\hline 5-O-cafeoilquinico & 1867594 & 0 & 1684792 & 2594656 & 10563927 & 141327 & 0 & 11127292 & 0 & 0 & 24886112 \\
\hline resveratrol & 0 & 0 & 9781,333 & 0 & 0 & 230230 & 347036 & 0 & 269733 & 0 & 0 \\
\hline pteroestilbeno & 0 & 0 & 0 & 772337 & 29253 & 0 & 131885 & 0 & 0 & 0 & 0 \\
\hline Apigenina & 0 & 0 & 5282,667 & 0 & 0 & 47951 & 55232 & 26371164 & 432953 & 4523560 & 0 \\
\hline catequina & 0 & 0 & 6275 & 2450051 & 0 & 1568970 & 1431070 & 152536715 & 3340108 & 40531371 & 0 \\
\hline malonato de dietila & 15716076 & 18375607 & 6259354 & 11908245 & 38713279 & 813377 & 1130448 & 40465699 & 1339151 & 9101858 & 9316329 \\
\hline$p$-coumarato de etila & 6589016 & 4154444 & 1683249 & 5715724 & 3494877 & 114120 & 141521 & 9581838 & 447482 & 3855233 & 3637326 \\
\hline galato de metila & 75590 & 1827184 & 101775,7 & 0 & 29145 & 766634 & 652375 & 0 & 0 & 0 & 3848466 \\
\hline Tirosol & 0 & 0 & 0 & 0 & 2029118 & 238748 & 191790 & 28578511 & 669940 & 7196871 & 0 \\
\hline
\end{tabular}

PERIÓDICO TCHÊ QUÍMICA•www.periodico.tchequimica.com• Vol. 15 N. 29.

- ISSN 1806-0374 (impresso) • ISSN 1806-9827 (CD-ROM) • ISSN 2179-0302 (meio eletrônico) 
Continuação. Tabela 3.

\begin{tabular}{|c|c|c|c|c|c|c|c|c|c|c|c|}
\hline \multirow{2}{*}{ Substâncias } & \multicolumn{11}{|c|}{ Abundância } \\
\hline & DC & DG & EP & GA & GB & GD & GD_B & SA_isaB & SA_isaM & SA_ita_B & SA_mosc_B \\
\hline acido fosforico & 2377009 & 131323179 & 152994635 & 807372 & 92878325 & 114270976 & 76364384 & 455333 & 455333 & 151544928 & 112767179 \\
\hline acido tartarico & 662411 & 1987336 & 64257531 & 10723560 & 17649921 & 26465027 & 1671565 & 67926 & 67926 & 56183579 & 47190107 \\
\hline acido málico & 769705 & 413738923 & 3975763 & 6217445 & 5087845 & 396927616 & 42687773 & 263769 & 263769 & 340287616 & 174503872 \\
\hline acido carbonico & 0 & 0 & 405857 & 192165 & 0 & 1992992 & 0 & 610473 & 606356 & 0 & 0 \\
\hline ácido propiônico & 0 & 3999014 & 4063460 & 291200 & 5050650 & 4485868 & 1499551 & 0 & 0 & 4090787 & 0 \\
\hline acido citrico & 235023 & 4838529 & 3258470 & 302300 & 5908676 & 4904763 & 7525249 & 1319176 & 1319176 & 2764987 & 1916775 \\
\hline acido succinico & 0 & 6572141 & 2693475 & 259896 & 13085773 & 5123049 & 2118760 & 48891 & 48891 & 2024844 & 1982805 \\
\hline acido acetico & 68518 & 4380021 & 6831575 & 472906 & 7526071 & 6171544 & 2514884 & 0 & 0 & 5679775 & 3869384 \\
\hline acido ascorbico & 124952 & 5859413 & 8414148 & 493221 & 10429829 & 9288212 & 3245652 & 11863 & 11863 & 7283019 & 5224982 \\
\hline acido $p$-coumarico & 106480 & 5599480 & 10408506 & 489542 & 14609703 & 6047141 & 1976003 & 9378 & 9378 & 6322637 & 1566968 \\
\hline acido cafeico & 144647 & 27208619 & 2739072 & 2277777 & 3161246 & 2722423 & 1051898 & 63336 & 63336 & 2421675 & 1640284 \\
\hline acido quinico & 0 & 4838529 & 3258470 & 302300 & 6198166 & 4904763 & 7525249 & 12771 & 12771 & 8062391 & 1916775 \\
\hline acido trans -fertarico & 662411 & 21750016 & 2692126 & 543098 & 61124549 & 9940201 & 9695183 & 9378 & 9378 & 3229249 & 47190107 \\
\hline ácido ferúlico & 0 & 21750016 & 2692126 & 10723560 & 61124549 & 24203533 & 9695183 & 67926 & 67926 & 3229249 & 47190107 \\
\hline ácido malonico & 141283 & 2278608 & 0 & 266756 & 5210837 & 0 & 1006829 & 92136 & 92136 & 0 & 0 \\
\hline ácido aspártico & 244485 & 17296912 & 10194731 & 261360 & 8950100 & 17092620 & 1823244 & 10971 & 10971 & 14669603 & 7360421 \\
\hline acido galico & 0 & 2919176 & 2431022 & 406049 & 6794092 & 3743760 & 1195370 & 10357 & 0 & 1916834 & 0 \\
\hline ácido cinâmico & 376942 & 4085792 & 64257531 & 10723560 & 17649921 & 5207389 & 1671565 & 32555 & 203779 & 3820513 & 0 \\
\hline ácido coutarico & 0 & 4183995 & 2712553 & 10723560 & 4831524 & 5431589 & 790443 & 67926 & 67926 & 2034055 & 2509734 \\
\hline ácido sinapico & 0 & 0 & 0 & 200523 & 3990226 & 0 & 0 & 250070 & 27742 & 0 & 1388068 \\
\hline ácido vanilico & 0 & 0 & 0 & 0 & 0 & 0 & 0 & 11896 & 0 & 0 & 0 \\
\hline ácido trans-caftárico & 0 & 2314819 & 0 & 0 & 0 & 0 & 0 & 9157 & 9157 & 0 & 0 \\
\hline ácido cis -4-coumarico & 0 & 4790289 & 2712553 & 489542 & 9000172 & 6648357 & 3288353 & 14854 & 14854 & 2034055 & 2845111 \\
\hline L-asparagina & 769705 & 3029160 & 3975763 & 419970 & 5087845 & 2878338 & 42687773 & 263769 & 263769 & 2433561 & 1246847 \\
\hline L-glutamina & 217945 & 1987336 & 11090287 & 914980 & 4024860 & 12036597 & 4694900 & 15681 & 15681 & 10507706 & 7259035 \\
\hline$L$ - lisina & 0 & 1987336 & 0 & 3185544 & 4024860 & 0 & 0 & 0 & 0 & 0 & 0 \\
\hline$L$-valina & 343308 & 5689325 & 0 & 0 & 3820176 & 7565829 & 0 & 0 & 0 & 0 & 0 \\
\hline$D$-glucose & 608718 & 27208619 & 42661813 & 2277777 & 48718539 & 42728064 & 1600628 & 63336 & 63336 & 37539107 & 25517859 \\
\hline L-prolina & 244485 & 3029160 & 0 & 419970 & 0 & 2878338 & 0 & 81212 & 81212 & 2433561 & 1246847 \\
\hline L-metionina & 769705 & 2407480 & 2639389 & 453839 & 4024860 & 0 & 1408591 & 263769 & 263769 & 2360311 & 1972543 \\
\hline L-triptofano & 0 & 0 & 0 & 0 & 0 & 2551931 & 837470 & 0 & 3159971 & 0 & 0 \\
\hline 5-O-cafeoilquinico & 68484 & 2848294 & 0 & 0 & 6352601 & 2826798 & 793790 & 0 & 0 & 0 & 0 \\
\hline resveratrol & 125638 & 0 & 0 & 0 & 0 & 0 & & 10774 & 77575 & 106996 & 4743540 \\
\hline pteroestilbeno & 222480 & 0 & 513627 & 0 & 0 & 0 & 0 & 0 & 0 & 0 & 2270503 \\
\hline Apigenina & 0 & 6572141 & 0 & 0 & 0 & 0 & 910535 & 10905 & 0 & 2024844 & 0 \\
\hline Catequina & 0 & 27208619 & 42661813 & 0 & 0 & 0 & 0 & 13935 & 0 & 0 & 1342361 \\
\hline galato de metila & 258839 & 0 & 0 & 0 & 0 & 0 & 0 & 86438 & 86438 & 0 & 0 \\
\hline p-coumarato de etila & 0 & 4838529 & 3258470 & 302300 & 7200135 & 4904763 & 7525249 & 12771 & 12771 & 2764987 & 1916775 \\
\hline malonato de dietila & 528738 & 8207656 & 11532785 & 1811111 & 13559384 & 13798979 & 4564038 & 18298 & 18298 & 9937167 & 7361931 \\
\hline Tirosol & 80596 & 5689325 & 7541499 & 3219157 & 9000172 & 1992992 & 3288353 & 22289 & 74730 & 7038343 & 47190107 \\
\hline
\end{tabular}

PERIÓDICO TCHÊ QUÍMICA•www.periodico.tchequimica.com• Vol. 15 N. 29. 
Continuação. Tabela 3.

\begin{tabular}{|c|c|c|c|c|c|c|c|c|c|c|c|}
\hline \multirow{2}{*}{ Substâncias } & \multicolumn{11}{|c|}{ Abundância } \\
\hline & MT & MV & $\mathrm{PE}$ & PE_B & PG & PZ & SB & SI & SN & SP & UV \\
\hline acido fosforico & 3033854 & 1138737 & 184025749 & 177958869 & 177748256 & 63297973 & 67071515 & 57296629 & 116386389 & 10019937 & 115049611 \\
\hline acido tartarico & 914456 & 298125 & 96171787 & 72375936 & 37895027 & 5399938 & 27625355 & 18412580 & 41240088 & 3096804 & 64839659 \\
\hline acido carbonico & 932515 & 944128 & 0 & 0 & 0 & 0 & 0 & 0 & 0 & 0 & 0 \\
\hline acido málico & 991862 & 422803 & 198159253 & 285889365 & 277166144 & 368019627 & 186771563 & 184048917 & 291749376 & 341605 & 528537771 \\
\hline acido citrico & 3805974 & 4074804 & 3124237 & 3763986 & 3891735 & 7953394 & 4681777 & 3894881 & 2252231 & 135117 & 8546573 \\
\hline ácido propiônico & 0 & 0 & 3951093 & 4672309 & 4285667 & 4231042 & 3317781 & 2960383 & 3838674 & 0 & 1370489 \\
\hline acido succinico & 0 & 0 & 1998265 & 6114095 & 2973377 & 9452278 & 6046972 & 6812175 & 4604826 & 0 & 2504162 \\
\hline acido acetico & 83581 & 0 & 6241681 & 6642979 & 6458445 & 4638100 & 5295985 & 4647222 & 5037321 & 1878154 & 2888867 \\
\hline acido ascorbico & 162695 & 79457 & 7402505 & 8925659 & 8501401 & 6955626 & 7708847 & 7690043 & 6195705 & 328672 & 3702139 \\
\hline ácido p-coumarico & 129163 & 88675 & 7586214 & 4632301 & 8831130 & 7397462 & 10350829 & 6318167 & 8242679 & 197307 & 4098121 \\
\hline ácido cafeico & 777609 & 370371 & 2356799 & 2672777 & 2683612 & 32593789 & 2497447 & 2488608 & 28262064 & 1663706 & 16481816 \\
\hline ácido quinico & 0 & 0 & 3124237 & 3763986 & 3891735 & 7953394 & 4681777 & 3894881 & 2252231 & 135117 & 8546573 \\
\hline ácido trans-fertarico & 914456 & 298125 & 12428217 & 17069745 & 13505741 & 52561109 & 27625355 & 8172225 & 34349021 & 0 & 3259906 \\
\hline ácido ferúlico & 914456 & 298125 & 10676113 & 17069745 & 13505741 & 52561109 & 29604307 & 22462179 & 34349021 & 368695 & 3259906 \\
\hline ácido aspártico & 295022 & 191065 & 8346379 & 12058475 & 11947383 & 15514704 & 7876605 & 7873202 & 12450143 & 145060 & 22370472 \\
\hline ácido malonico & 139379 & 168071 & 0 & 2341647 & 0 & 4571289 & 2736668 & 2642827 & 2833114 & 0 & 1643435 \\
\hline ácido sinápico & 0 & 0 & 0 & 0 & 0 & 25139100 & 0 & 0 & 0 & 132293 & 0 \\
\hline acido gálico & 0 & 0 & 2630850 & 8162487 & 7339621 & 1282100 & 3439912 & 3956927 & 3694896 & 0 & 1533940 \\
\hline ácido cinâmico & 914456 & 894376 & 96171787 & 217127808 & 113685080 & 72178744 & 27625355 & 4795871 & 41240088 & 3096804 & 1839578 \\
\hline ácido siringico & 0 & 0 & 0 & 3465944 & 0 & 0 & 0 & 0 & 0 & 0 & 0 \\
\hline ácido coutarico & 914457 & 298125 & 2389681 & 3607901 & 4946112 & 8262047 & 5004696 & 5332045 & 4458329 & 3096804 & 3640168 \\
\hline ácido cis-4-coumarico & 169189 & 74759 & 3557768 & 3607901 & 2061028 & 8262047 & 5004696 & 2247115 & 5414800 & 341605 & 3640168 \\
\hline ácido trans-caftárico & 0 & 0 & 0 & 0 & 2418178 & 4806616 & 0 & 2602746 & 0 & 0 & 2239860 \\
\hline$L$-asparagina & 295022 & 422803 & 2301950 & 3141138 & 6929336 & 5615338 & 4788938 & 4722295 & 2142257 & 860431 & 3968917 \\
\hline L-glutamina & 284130 & 137225 & 9990540 & 12073644 & 11437400 & 9308720 & 1899780 & 2155490 & 3198546 & 539905 & 1421170 \\
\hline$L$-lisina & 0 & 0 & 0 & 0 & 0 & 0 & 1899780 & 2155490 & 3198546 & 0 & 1421170 \\
\hline L-valina & 0 & 0 & 0 & 11383625 & 0 & 0 & 0 & 0 & 0 & 0 & 0 \\
\hline$D$-glucose & 777609 & 370371 & 2050079 & 41352685 & 42376832 & 32593789 & 38809773 & 38307792 & 28262064 & 1663706 & 16481816 \\
\hline L-prolina & 295022 & 191065 & 0 & 3141138 & 0 & 0 & 0 & 0 & 2142257 & 0 & 3968917 \\
\hline$L$-metionina & 991862 & 422803 & 3983560 & 3046702 & 0 & 0 & 1899780 & 2155490 & 3198546 & 128440 & 2714894 \\
\hline$L$-arginina & 0 & 0 & 0 & 0 & 0 & 17626078 & 0 & 0 & 0 & 0 & 0 \\
\hline$L$-leucina & 0 & 142741 & 0 & 0 & 0 & 0 & 0 & 0 & 0 & 0 & 0 \\
\hline L-triptofano & 0 & 0 & 0 & 0 & 0 & 0 & 0 & 0 & 0 & 0 & 2548352 \\
\hline 5-O-cafeoilquinico & 0 & 62800 & 0 & 0 & 0 & 6353685 & 1993212 & 3907728 & 2457629 & 0 & 1450526 \\
\hline resveratrol & 116529 & 279984 & 0 & 0 & 0 & 0 & 0 & 0 & 0 & 0 & 0 \\
\hline pteroestilbeno & 0 & 219638 & 0 & 0 & 227972 & 0 & 0 & 0 & 0 & 188156 & 0 \\
\hline Apigenina & 0 & 0 & 1998265 & 18342284 & 8920131 & 28356834 & 0 & 12425655 & 4604826 & 138856 & 0 \\
\hline Catequina & 777609 & 0 & 0 & 0 & 0 & 0 & 0 & 0 & 0 & 0 & 0 \\
\hline malonato de dietila & 635731 & 362862 & 10445617 & 11192249 & 11133893 & 9980141 & 10151768 & 11189041 & 8639457 & 1023376 & 5722007 \\
\hline$p$-coumarato de etila & 0 & 0 & 3124237 & 3763986 & 3891735 & 7953394 & 4681777 & 3894881 & 2252231 & 135117 & 8546573 \\
\hline galato de metila & 242031 & 270660 & 0 & 0 & 0 & 0 & 0 & 0 & 0 & 229748 & 0 \\
\hline manitol & 0 & 0 & 0 & 0 & 0 & 0 & 2107657 & 0 & 0 & 0 & 0 \\
\hline Tirosol & 557983 & 224279 & 6422621 & 22898894 & 23153564 & 17300734 & 7193702 & 6634847 & 5414800 & 0 & 0 \\
\hline
\end{tabular}

Os termos AL, AU etc. são os códigos referentes aos sucos de uva (vide Tabela 1). Os valores de abundância referem-se às médias das áreas relativas das triplicatas de extração. 
Tabela 4. Substâncias com seus respectivos códigos para análise dos componentes principais (PCA)

\begin{tabular}{lc|lc}
\hline \multicolumn{1}{c}{ Substância } & Código para PCA & \multicolumn{1}{c}{ Substância } & Código para PCA \\
\hline acido fosfórico & C1 & - valina & C23 \\
acido tartárico & C2 & $D$ - glucose & C24 \\
acido málico & C3 & L- prolina & C32 \\
acido cítrico & C4 & L- metionina & C33 \\
acido succínico & C5 & L-tirosina & C34 \\
acido acético & C7 & L- leucina & C40 \\
acido ascórbico & C8 & L-triptofano & C48 \\
ácido p-coumarico & C9 & L-arginina & C54 \\
ácido cafeico & C12 & L- glutamina & C21 \\
ácido quinico & C13 & L-asparagina & C19 \\
ácido trans-fertarico & C16 & resveratrol & C44 \\
ácido ferúlico & C17 & pteroestilbeno & C50 \\
ácido aspártico & C20 & Apigenina & C42 \\
ácido malonico & C27 & catequina & C47 \\
ácido siringico & C31 & coniferina & C53 \\
ácido propionico & C36 & Tirosol & C41 \\
acido galico & C45 & manitol & C56 \\
ácido cinâmico & C46 & 5-O-cafeoilquinico & C37 \\
ácido sinapico & C59 & 4-O-cafeoilquinico & C58 \\
ácido coutarico & C39 & galato de metila & C35 \\
ácido trans-caftárico & C30 & malonato de dietila & C25 \\
ácido cis-4-coumarico & C28 & $p$-coumarato de etila & C18 \\
$L-$ lisina & C22 & & \\
\hline
\end{tabular}




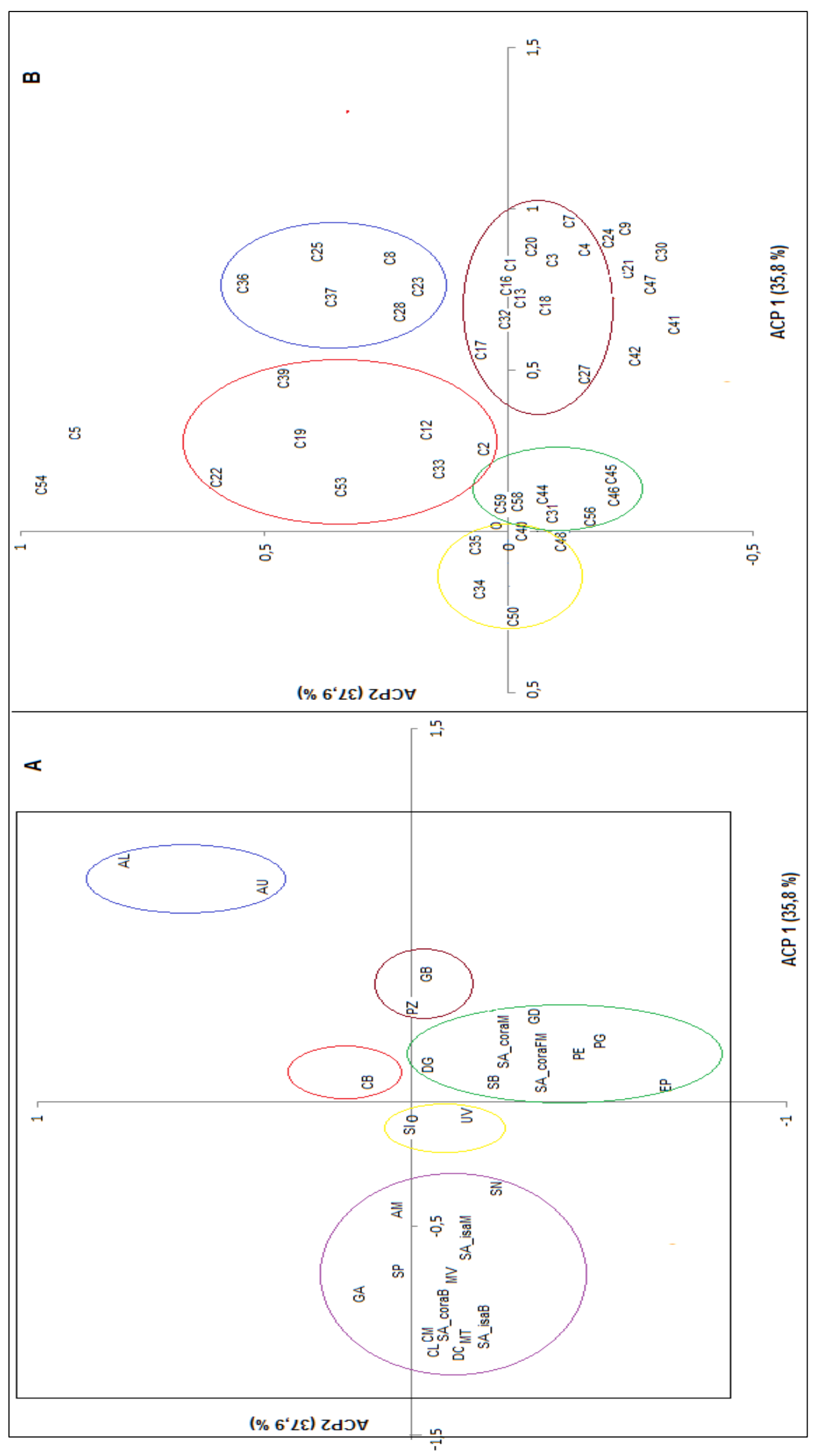

Figura 1. (A) Gráfico de scores dos sucos de uva tintos, (B) Gráfico de loadings com os compostos não-voláteis identificados, com formação de agrupamentos conforme as cores observadas nos gráficos $(A)$ e $(B)$. 


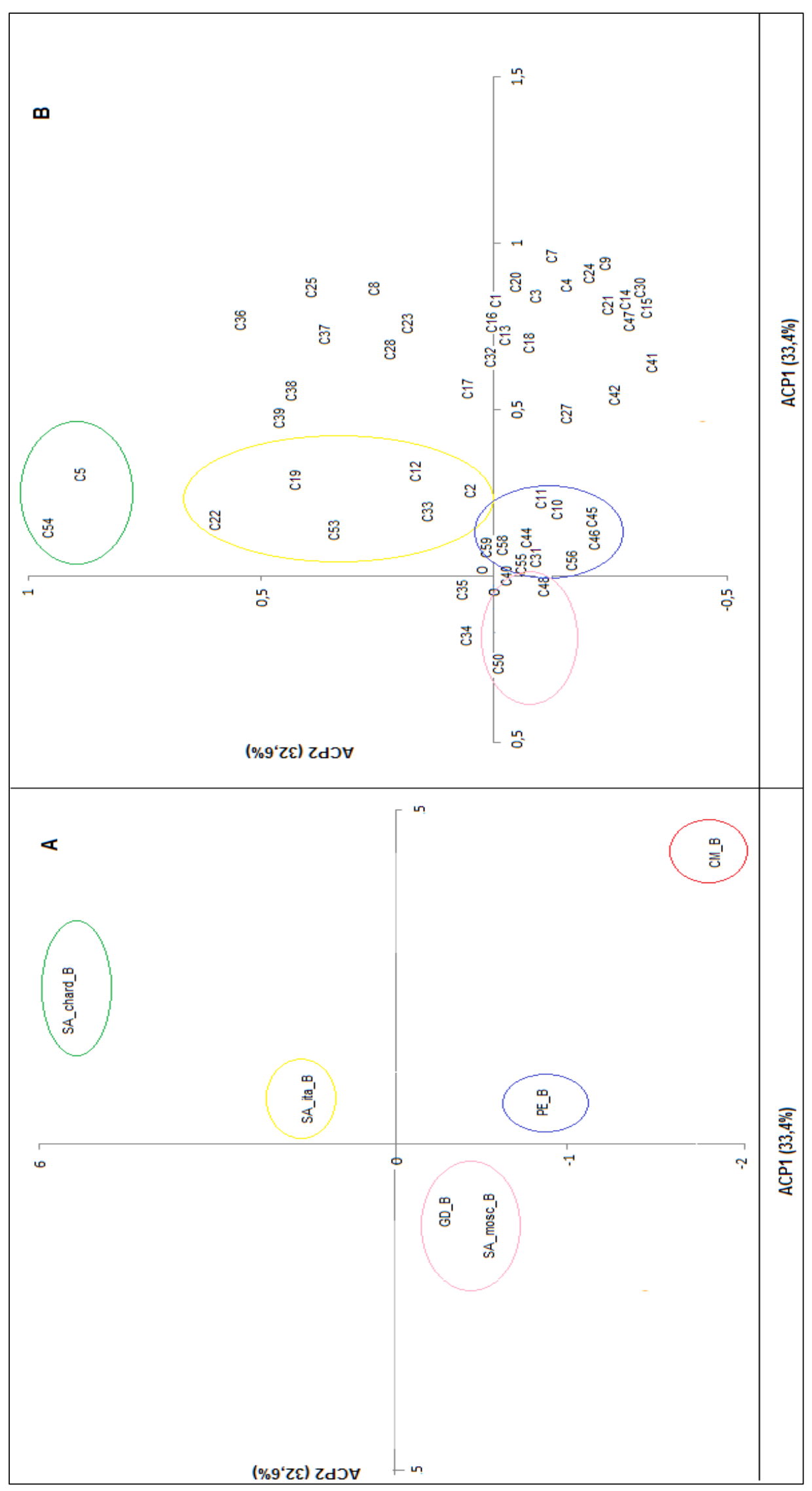

Figura 2. (A) Gráfico de scores dos sucos de uva brancos, (B) Gráfico de loadings com os compostos não-voláteis identificados, com formação de agrupamentos conforme as cores observadas nos gráficos $(A)$ e $(B)$. 